\title{
Exposición a ruidos por el ejercicio profesional en docentes odontólogos
}

Ferrando $K^{1}$

Chirife $T^{2}$

Jacquett $N^{3}$

\section{Resumen}

Objetivo: determinar la exposición a ruidos por el ejercicio profesional en docentes odontólogos de una Universidad privada de Asunción en el año 2010. Material y métodos: el muestreo fue no probabilístico de casos consecutivos, dentro una población de 90 docentes odontólogos de una Universidad Privada de Asunción, participaron en el estudio de forma voluntaria un total de 70, cuyas edades oscilaron entre 30 y 87 años de edad. Resultados: En su mayoría los profesionales encuestados ejercen su profesión en forma privada, por lo que podrían tener menor posibilidad de padecer problemas auditivos, debido a que tienen menos horas de exposición a ruidos, en comparación con los que ejercen la profesión en el ámbito privado y público. Los docentes son diestros en su mayor porcentaje, teniendo más po- sibilidades de tener afectado el oído derecho que el izquierdo. El 95,7\% de los profesionales no utiliza ningún tipo de protector auditivo durante su práctica odontológica, también el predominio del sexo femenino dentro de la población encuestada y la mayor cantidad de odontólogos con edades comprendidas entre 30 y 40 años equivalentes a un $40 \%$ del total de la población. Con respecto a la percepción auditiva el $90 \%$ de los profesionales respondió que escuchaba bien, mientras que el 1,4\% respondió que usaba algún tipo de protector auditivo durante la práctica profesional. Conclusión: Los datos presentados a continuación resaltan que los profesionales docentes están expuestos a diferentes tipos de ruidos que con el tiempo podrían ocasionar pérdida auditiva.

Palabras Claves: pérdida auditiva, práctica profesional.

Artigo Original

\section{Exposiçâo a ruídos pelo exercício profissional em professores de odontologia}

\section{Resumo}

Objetivo: determinar a exposição a ruídos pelo exercício profissional em docentes odontólogos de uma Universidade privada de Assunção no ano de 2010. Material e métodos: a amostragem foi não probabilística de casos consecutivos. Em uma população de 90 docentes odontólogos de

\footnotetext{
${ }^{1}$ Alumna del posgrado de Odontopediatría de la Facultad de Odontología Universidad Autónoma del Paraguay (UAP).

${ }^{2}$ Coordinadora de Posgrados de Odontopediatría de la Facultad de Odontología Universidad Autónoma del Paraguay (UAP).

${ }^{3}$ Especialista. Máster en Odontopediatría de la Facultad de Odontología Universidad Autónoma del Paraguay (UAP).
} 
uma Universidade Privada de Assunção, participaram do estudo, voluntariamente, um total de 70 docentes, com idade entre 30 e 87 anos. Realizou-se um estudo transversal, observacional, descritivo. Resultados: em sua maioria os profissionais interrogados exercem sua profissão em forma privada, pelo que poderiam ter menor possibilidade de sofrer problemas auditivos, já que têm menos horas de exposição a ruídos, em comparação com os que exercem a profissão em o âmbito privado e público. Os docentes são destros em sua maior percentagem, tendo maior possibilidade de ter afetado o ouvido direito que o esquerdo. 95,7\% dos profissionais não utiliza nenhum tipo de protetor au- ditivo durante a prática odontológica, também houve predomínio do sexo feminino e a maior parte, aproximadamente $40 \%$, do total de entrevistados estava na faixa etária dos 30 a 40 anos de idade. Com respeito à percepção auditiva, 90\% dos profissionais respondeu que escutava bem, enquanto $1,4 \%$ respondeu que usava algum tipo de protetor auditivo durante a prática profissional. Conclusão: os dados apresentados a seguir ressaltam que os profissionais docentes estão expostos a diferentes tipos de ruídos que com o tempo poderiam ocasionar perda auditiva.

Palavras Chaves: perda auditiva, prática profissional.

\section{Original article}

\section{Noise exposures in professional practice in dentistry teachers}

\section{Abstract}

Objetive: assessing noise exposure for the dental faculty practice in a private University of Asuncion in 2010." The sampling was not probabilistic consecutive cases in a population of 90 teachers from a private university dentists Asuncion, participated in the study voluntarily a total of 70, whose ages ranged between 30 and 87 years of age. Results: Most respondents professionals practicing in private, so they may be less likely to have hearing problems, because they have fewer hours of noise exposure compared to those practicing in the private and public. That teachers are skilled at its highest percentage, with more likely to have affected the right ear than the left. That $95.7 \%$ of professionals do not use any hearing protector for your dental practice, it also emphasizes the predominan- ce of women in the survey population and the largest number of dentists aged between 30 and 40 years equivalent to $40 \%$ of the total population. With regard to auditory perception, $90 \%$ of practitioners responded that he listened well, while $1.4 \%$ said they used some type of hearing protection during practice. Conclusion: The data presented below highlight the professional teachers are exposed to different types of noises that could eventually lead to hearing loss.

Keys words: hearing loss, practice professional.

\section{Introducción}

El aumento de las fuentes productoras de ruido está afectando la calidad de vida de los profesionales, acelerando el deterioro del aparato auditivo. (1) 
El odontólogo está expuesto diariamente al ruido producido por los equipos, entre ellos la turbina de alta rotación, compresores de aire, eyectores de saliva, etc., y están propensos a la disminución de su capacidad auditiva, ya que varios de los instrumentos utilizados dentro del consultorio dental emiten sonidos agudos y de alta intensidad que sobrepasan los limites de seguridad de la salud auditiva del odontólogo. $(2,3,4)$.

Se estima que un tercio de la población mundial y el $75 \%$ de los habitantes de ciudades industriales padecen de algún grado de sordera o pérdida auditiva causada por exposición a sonidos de alta intensidad. La OPS refiere una prevalencia promedio de hipoacusia del $17 \%$ para América Latina en trabajadores con jornadas de 8hs diarias, durante 5 días a la semana con una exposición que varía entre 10 y 15 años. $(5,6)$

En una prueba realizada con 137 odontólogos y 80 médicos. Al comparar los resultados se evidenció que los médicos tienen mejores niveles de audición, además se encontró que el oído izquierdo de los odontólogos diestros mostró un nivel menor de pérdida auditiva, relacionada presumiblemente a su mayor distancia con la fuente de sonido. $(7,8)$

Existen indicios de que muchas veces, el profesional expuesto a este tipo de riesgo laboral se acostumbra al ruido y no es consciente de que puede estar causando un daño irreparable en su audición. La pérdida de la audición es una combinación de la edad y el uso de turbina. La sordera está relacionada con el oído del lado en que se utiliza la pieza de mano. (9)

El ruido también provoca estrés, fatiga, pérdida de capacidad de reacción y comunicación, que pueden ser causantes de accidentes y contribuir a la aparición de problemas circulatorios, digestivos y nerviosos. (9)

Está demostrado que el uso de piezas de mano de alta velocidad, cuyos motores realizan un promedio de 300.000 a 400.000 revoluciones por minuto, está relacionado con la posibilidad de sordera inducida por ruido. (9)

Entre los odontólogos, se encontró niveles similares de daño en la audición en el grupo de especialistas, al compararlos con los odontólogos de práctica general. (9)

En el caso de no poder obtener valores aceptables y seguros de ruido a nivel ambiental, está indicado el uso de protectores auditivos, estos dispositivos se utilizan desde 1945 y desde entonces se han diseñado muchas variedades. (10)

Se recomienda el uso de tapones de oídos con el fin de atenuar la intensidad y frecuencia del ruido. Además establecen en lo posible la distancia entre el odontólogo y el paciente. (11)

Entre las formas de prevenir la pérdida de audición podemos citar las siguientes, conocer los ruidos que pueden causar daños y son aquellos que están por encima de los 80 decibeles, usar orejeras $\mathrm{u}$ otros dispositivos protectores de la audición cuando se realice una actividad que involucre sonidos que dañan la audición, estar alerta a los ruidos peligrosos que se producen en el consultorio odontológico, realizar exámenes médicos periódicos (11).

La importancia de la audición en la vida diaria para realizar las diferentes actividades cotidianas, fue el motivo principal por el cual se realizó este trabajo con el objetivo de "Evaluar la exposición a ruidos por el ejercicio profesional en docentes odontólogos, de una Universidad privada de Asunción en el año 2010”. Este estudio 
podría servir para incentivar futuros programas a fin de prevenir la pérdida auditiva de profesionales odontólogos de nuestro país; y también busca contribuir y ampliar los conocimientos en este campo.

\section{Objetivo General}

Evaluar la exposición a ruidos por el ejercicio profesional en docentes odontólogos, de una Universidad Privada de Asunción en el año 2010.

\section{Objetivos específicos}

1. Clasificar los datos de acuerdo a la edad, género y formación profesional.

2. Indagar el nivel de exposición a ruidos por el ejercicio profesional.

3. Identificar el nivel de ruido percibido dentro del ejercicio profesional. privado y público.

4. Conocer sobre antecedentes familiares, enfermedades, medicamentos relacionados con la predisposición de la pérdida auditiva.

5. Indagar sobre el uso de protectores auditivos por parte de los docentes.

\section{Material y Método}

El diseño que se utilizó para este trabajo fue observacional, descriptivo, de corte transverso. El muestreo fue no probabilístico de casos consecutivos, dentro una población de 90 docentes odontólogos de una Universidad Privada de Asunción, participaron en el estudio de forma voluntaria un total de 70, cuyas edades oscilaron entre 30 y 87 años de edad.
El reclutamiento se solicitó por medio de una carta de permiso a la decana de una Universidad Privada de Asunción, el levantamiento de datos se realizo a través de un cuestionario que se le entrego a cada docente odontólogo con más de 10 años de ejercicio profesional.

- Criterios de inclusión: docentes odontólogos que tengan como mínimo diez años de ejercicio profesional, docentes odontólogos de una Universidad Privada que ejercen la docencia en el año 2010, docentes odontólogos que acepten participar en el estudio.

- Criterios de exclusión: Docentes odontólogos que tengan alguna afección auditiva congénita o adquirida durante la infancia, o por causas ajenas a la profesión, docentes odontólogos con permiso en el año de toma de datos, docentes odontólogos ausentes el día del levantamiento de datos.

Fue aplicado un cuestionario diseñado para el levantamiento de datos (ver apéndice I). Con las siguientes variables, dentro de datos generales: género, formación profesional; odontólogo ó odontólogo especialista, área de especialización, años de ejercicio profesional, tipo de práctica profesional; privada, pública o ambas, tiempo dedicado a la práctica odontológica (privada o institucional) en días por semana, tiempo dedicado en horas por día a la práctica odontológica (privada o institucional), postura usual en su práctica odontológica, mano utilizada durante la práctica odontológica; derecha, izquierda, ambas.

Dentro de datos específicos: antecedentes familiares, tóxicos laberínticos, enfermedades generales con afectación ótica, antecedentes otológicos, percepción auditiva.

El levantamiento de datos se realizó a través de un encuestador calibrado, utilizando como re- 
cursos materiales un cuestionario diseñado para levantamiento de datos.

En el estudio se cumplieron con los criterios éticos en cuanto a respeto, beneficio y justicia para ésta investigación, solicitando autorización por medio de cartas de permiso al decano, para el levantamiento de datos a través del cuestionario a profesionales docentes odontólogos. Se entregó el cuestionario a aquellos profesionales odontólogos que hayan aceptado participar del estudio. Se respetó la autonomía de los profesionales docentes odontólogos en la participación del estudio y se mantuvo la confidencialidad de los datos. Se respetó la identidad de la universidad.

\section{Resultados}

Los resultados de la presente investigación que buscó evaluar el nivel de exposición a ruidos por el ejercicio profesional, estuvo comprendida por un total de 70 odontólogos seleccionados de acuerdo a los criterios de inclusión y exclusión establecidos en la metodología.

La mayoría de los profesionales encuestados manifestó que no utiliza ningún tipo de protector auditivo y el procedimiento más realizado fue el de Operatoria Dental (33\%) dentro de la práctica privada $(60 \%) 5$ días a la semana (67\%), 4 a 6 horas por día $(64,3 \%)$.

E171,4\% no tuvo ningún familiar con problemas otológicos, mientras que el 18,6\% respondió que su padre tenía o tuvo problemas otológicos.

El 94,3\% de los encuestados respondió que no consume ningún medicamento del tipo ototóxico.

En cuanto a la percepción auditiva se les realizó algunas preguntas a los profesionales dando como resultado: Que el $85,7 \%$ oye bien, mien-
Cuadro I: Distribución del porcentaje de profesionales de acuerdo al género. N: 70.

\begin{tabular}{|c|c|c|}
\hline Femenino & 37 & $52,9 \%$ \\
\hline Masculino & 33 & $47,1 \%$ \\
\hline Total & 70 & $100 \%$ \\
\hline
\end{tabular}

Cuadro II: Clasificación según la formación profesional. N: 70 .

\begin{tabular}{|c|c|c|}
\hline & Frecuencia & Porcentaje \\
\hline Odontólogo & 11 & $15,7 \%$ \\
\hline Especialista & 59 & $84,3 \%$ \\
\hline Total & 70 & $100 \%$ \\
\hline
\end{tabular}

tras que el 14,3\% respondió que no oye bien, del $65 \%$ respondió que no necesita hacerse repetir, mientras que el $35 \%$ de los encuestados respondió que debe aumentar el volumen de la televisión, al 91,4\% le molestan los ruidos intensos.

En el cuadro I se puede observar la distribución de frecuencia de profesionales de acuerdo al género, el 52,9\% eran del sexo femenino y el 47,1\% del sexo masculino.

En el cuadro II el 84, 3\% de los encuestados tenían un título de especialista a diferencia de los que tenían, solo el título de odontólogo con un $15,7 \%$.

En el cuadro III vemos que la mayor frecuencia de edad de los profesionales oscilaron entre los 30 a 40 años con un $40 \%$.

El 71,4\% de los profesionales no presenta antecedentes otológicos, mientras que el 18,6 \% padece tinnitus. (Cuadro IV) 
Cuadro III: Clasificación de acuerdo a la edad de los profesionales. N: 70.

\begin{tabular}{|c|c|c|}
\hline Edades & Frecuencia & Porcentaje \\
\hline 30 a 40 años & 28 & $40,0 \%$ \\
\hline 40 a 50 años & 21 & $30,0 \%$ \\
\hline 50 a 60 años & 14 & $20,0 \%$ \\
\hline 70 a 80 años & 6 & $8,6 \%$ \\
\hline 80 a 90 años & 1 & $1,4 \%$ \\
\hline Totales & 70 & $100 \%$ \\
\hline
\end{tabular}

Cuadro IV: Antecedentes otológicos. N: 70.

\begin{tabular}{|c|c|c|}
\hline \multicolumn{3}{|c|}{ Antecedentes Otológicos } \\
\hline & $\mathbf{n}$ & $\%$ \\
\hline Tinnitus & 13 & 18,6 \\
\hline Zumbidos & 2 & 2,9 \\
\hline Vértigo & 4 & 5,7 \\
\hline Mareos & 1 & 1,4 \\
\hline Ninguno & 50 & 71,4 \\
\hline Totales (n: 70) & 70 & 100 \\
\hline
\end{tabular}

Cuadro V: Utiliza algún protector auditivo. N: 70.

\begin{tabular}{|c|c|c|}
\hline \multicolumn{2}{|c|}{$\begin{array}{r}\text { Utiliza algún protector auditivo en el consultorio } \\
\text { durante su práctica odontológica }\end{array}$} \\
\hline & $\mathrm{n}$ & $\%$ \\
\hline Sí & 1 & $1,4 \%$ \\
\hline No & 67 & $95,7 \%$ \\
\hline A veces & 2 & $2,9 \%$ \\
\hline Total & 70 & $100 \%$ \\
\hline
\end{tabular}

El 95,7\% correspondiente a 67 de los profesionales encuestados respondieron que no utilizan ningún protector auditivo durante la práctica odontológica (Cuadro V y Gráfico 1).

\section{Discusión y comentarios}

En este trabajo los profesionales odontólogos son en su mayoría diestros y realizan con mayor frecuencia durante su práctica trabajos en el área de operatoria dental, estos datos estarían relacionados a estudios en donde se concluyeron que el oído izquierdo de los odontólogos mostró un nivel menor de pérdida auditiva, relacionada presumiblemente a su mayor distancia a la fuente de sonidos $(6,7)$.

La OPS (7) refiere una prevalencia promedio de hipoacusia del $17 \%$ para América latina en trabajadores con jornadas de 8 hs diarias, durante 5 días a la semana con una exposición que varía entre 10 y 15 años, esto se relaciona con los datos encontrados en este trabajo donde los odontólogos trabajan de 4 a 6 hs los 5 días de la semana.

El cuadro y gráfico III, nos muestra que hay un predominio de profesionales con edades comprendidas entre 30 y 40 años, los cuales podrían formar parte de un grupo de riesgo, de presentar patologías auditivas debido a la exposición a

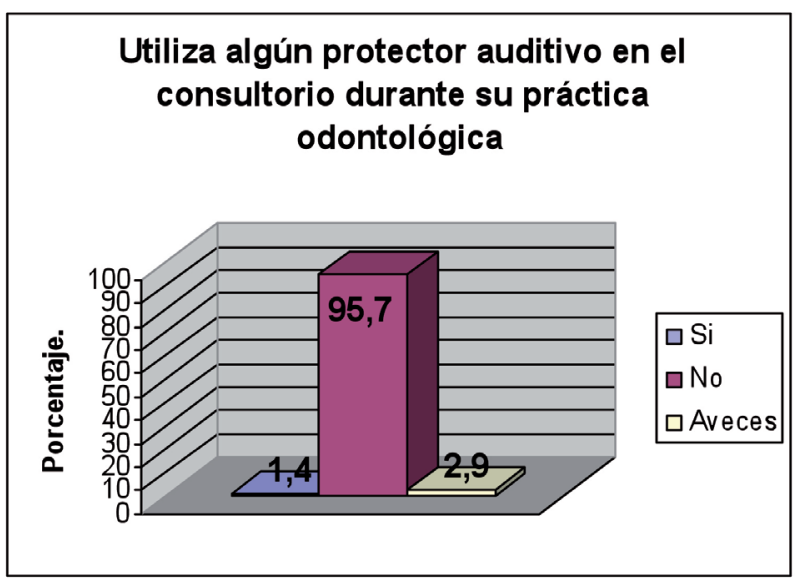

Gráfico 1. Utiliza algún protector auditivo. N: 70. 
ruido constante relacionado con la pieza de alta y baja velocidad, compresores, y además el ruido ambiental que se propicia durante al consulta, resultados que coinciden con la bibliografía consultada donde Flores Hernández y col. (35) coinciden de que el grupo de riesgo se encuentra entre los odontólogos entre 30 y 40 años.

En el cuadro XV vemos que el 95,7 \% de los profesionales no utiliza ningún tipo de protector auditivo durante su práctica odontológica, con respecto a esto Hinze y col. (11); recomiendan el uso de tapones o protectores de oído con el fin de atenuar la intensidad y frecuencia del ruido.

De acuerdo a los resultados obtenidos en este estudio se observa un porcentaje elevado de profesionales expuestos al ruido del consultorio dental, que no utilizan ningún tipo de protección auditiva, por lo que sería bueno evaluar, la necesidad de programas de prevención en donde se concientice a los profesionales a cerca de los daños que pueden sufrir en el aparato auditivo.

\section{Conclusiones}

De acuerdo a los resultados de este trabajo se puede concluir que:

- Los docentes odontólogos están expuestos a ruidos por el ejercicio profesional debido a que: la mayoría no utiliza protector auditivo, son diestros, estando expuestos diariamente; trabajando en su mayoría de 4 a 6 hs diarias, los 5 días de la semana, realizando el mayor porcentaje de profesionales encuestados procedimientos en el área de Operatoria Dental.

- Por otra parte la mayoría de los profesionales encuestados se encuentran con edades comprendidas entre 30 y 40 años, punto muy importante, ya que a partir de los 40 años se tiene más predisposición a tener trastornos auditivos.

\section{Recomendaciones}

Se considera de mucha importancia que los odontólogos estén al tanto de todos los efectos que tiene sobre la salud el ejercicio de la profesión odontológica. Ellos deben conocer las formas de prevenir estos efectos para mantener una buena salud.

Se les recomienda a los profesionales conocer cada uno de los efectos fisiológicos que pueden aparecer en el transcurso de la carrera. Uno de ellos es la disminución de la audición, ya que están expuestos al sonido excesivo que emiten diferentes instrumentos dentales que funcionan con motor o turbinas dentales.

Se ha visto que una forma fácil de prevenir es con la utilización de tapones para oídos u otros protectores auditivos, mientras se realice un trabajo que involucre sonidos excesivos como el de la turbina, eyector, etc. Para que no exista daño auditivo, el profesional no debe estar expuesto a sonidos de intensidad de más de $80 \mathrm{~dB}$ (decibeles).

Se recomienda conocer la intensidad de sonido a la que se está expuesto en el consultorio.

Por lo tanto seria de vital importancia informar y prevenir a nuestros profesionales acerca de los problemas auditivos que se puede adquirir debido a la práctica odontológica y incentivar el uso de protectores auditivos, si fuese posible desde el pregrado. 


\section{Referencias}

1-TOLOSA, F. Efectos de ruido sobre la salud. Traducción del curso académico en la Real Academia de Medicina de las Islas Balneares. 2003.Disponible en: www.google.com.mx fecha de acceso: 13 de abril de 2010.

2-GARBIN, A.; GARBIN, C.; FERREIRA, N. Evaluación de la incomodidad ocupacional: nivel del ruido de una clínica de graduación. Acta odontol. Venezuela, Enero. 2006. Disponible en: www.actaodontologica.com, fecha de acceso: 27 de abril de 2010.

3-WONG, J.; FRANCESCHI, T. Pérdida gradual de audición causada por el ruido emitido en el consultorio dental. Odontología Vital.2006.Disponible en: www.odontología-vital.ulatina.ac.cr.2006. fecha de acceso: 13 de abril de 2010.

4-ANGULO, S.; LÓPEZ M.; NAVA, A.; REBOLLAR, L.; ROJAS, S. Disminución auditiva provocada por el uso de la pieza de mano y de alta velocidad en odontólogos de la zona de Tlalnepantla.2006 Disponible en: www.odontologia.iztacala. unam.mx fecha de acceso: 11 de mayo de 2010.

5-OTERO, J. Pérdida de la audición en odontólogos.2002. Disponible en: www.Acta odontológica.com. fecha de acceso: 11 de mayo de 2010.

6-LANDEROS, E.; MARTINEZ, M.; MORA, A.; PATIÑO, E.; PEREZ, A. Enfermedades laborales en el cirujano dentista.2003.Disponible en: www.scielo.org fecha de acceso: 11 de mayo de 2010.

7-MALDONADO, G. Pérdida de audición en odontólogos.2005. Disponible en: www.actaodontologica.com, fecha de acceso: 27 de abril de 2010 .

8-Efectos nocivos del ruido en trabajadores de la construcción. Disponible en : www.odontopreven.com fecha de acceso: 20 de abril de 2010.

9- RIVAS, J.; ARIZA, H. Tratado de otología y audiología. Diagnóstico y tratamiento médico quirúrgico. 2da. Edición. Editorial AMOLCA; 2007; Pág. 469-504.

10- PIMENTEL, F. Efeito do ruido (ambiental sobre la salud) no homen e acordado. 2000. Disponible en: www.estudioodonto.com, fecha de acceso: 16 de marzo de 2010.

11- LANDEROS, E.; MARTINEZ, M.; MORA, A.; PATIÑO, E.; PEREZ, A. Enfermedades Laborales en el Cirujano Dentista. 2000. Disponible en: http: / / odontologia.iztacala.unam.mx/instrum fecha de acceso: 11 de mayo de 2010

12-ROUVIÉRE; DELMAS. Anatomía Humana. Editorial Masson. Buenos Aires. Volumen 1, 11 Edición 2005. Pág.415 462.

13-LATARJET, M; RUIZ, A. Anatomía humana. Editorial Médica Panamericana. Buenos Aires. Volumen I. Tercera Edición 1998: Pág. 481-507.

14- LATARJET, M; RUIZ, A. Anatomía humana. Editorial Médica Panamericana. Buenos Aires. Volumen I. Cuarta Edición 2004: Pág. 436-460.

15- VILLAMAYOR, S. JACQUETT N, CHIRIFE, M Nivel de conocimiento y práctica sobre métodos de protección auditiva en estudiantes del quinto año de facultades de odontología del Paraguay (tesis doctoral).Asunción: Universidad Autónoma del Paraguay.2011.

16- Efectos del Ruido Ambiental sobre la Salud.2003.Disponible en: www.scielo.brs.com fecha de acceso: 20 de abril de 2010.

17-MALDONADO, G. Presencia de sonidos que producen contaminación auditiva en las instalaciones clínicas intramurales de la facultad de odontología de la Universidad de San Carlos de Guatemala (Tesis doctoral). Universidad de San Carlos de Guatemala. Septiembre. 2005.

18- OTÁROLA MERINO, F.; OTÁROLA ZAPATA, F.; FINKELSTEIN, A. Ruido Laboral y su Impacto en Salud.2006. Disponible en: www.cienciay trabajo.cl./ polos/2006 fecha de acceso: 11 de mayo de 2010,

19- QUINTANA, J.; MACHIN, Y.; Bioseguridad en estomatología. 2009. Disponible en: www.cpicmha.sld.cu/hab/ vol13_2_07/hab15207.htm fecha de acceso: 25 de mayo de 2010.

20-GÓMEZ, P.; PÉREZ, B.; MENESES, A. Pérdidas auditivas relacionadas con la exposición a ruido en trabajadores de la construcción. Caracas. 2009. Disponible en: www.actaodontologica.com fecha de acceso: 25 de mayo de 2010. 
21- GOODHILL, V. El oído enfermedades, sordera y vértigo. Editorial Salvat. Barcelona.1986. Pág. 703 al 710,712 al 714. 22- JIMÉNEZ, L.; ALFARO, C. Lesiones posturales y mecánicas: una amenaza para el odontólogo. 2006. Disponible en: http: / / odontología-vital.ulatina.ac.cr fecha de acceso: 11 de mayo de 2010.

23- BARRANCOS MONEY, J.; BARRANCOS, P. Operatoria Dental. Editorial Panamericana. Buenos Aires. Cuarta Edición. 2006. Pág. 149 al 158.

24- BESS, H.; HUMES L. Fundamentos de audiología. Editorial El Manual Moderno S.A. de C.V.Mexico. Tercera Edición 2005. Pág.46.

25-SALESA, E.; PERELLÓ, E.; BONAVIDA, A. Tratado de Audiología. Editorial Masson S.A. Tercera Edición. Barcelona España 2005. Pág. 177-185.

26- ORO, C.; PIETROBON, L.; AGUILAR, M.; REGIS. G. Doencas de caráter ocupacional em cirurgioes - dentistas: uma revisao da literatura. XXVI ENEGEP -Fortaleza, CE, Brasil. 9 a 11 de outubro de 2006.

27- LIDA, N.; SANTOS. Eficacia de escolha do protetor auditivo pequeño, médio e grande em programa de conservacao auditiva. Rev.CEFAC. Out./ dez.2006, vo19, n.4

28-SOUSA, H.; MATTOS, U.; NUNES, F. Nivel de ruido producido por turbina de alta rotacao em consultorio odontológico. Rev. Bras. Odontol; 59 (3):169-72, maio-jun.2002.

29- BARRANCOS MONEY, J. Operatoria Dental. Editorial Panamericana. Buenos Aires.2000. Pág.126 al 139.

30-SANGUINETTI C.; SANGUINETTI L.; COL. Semiologia, semiotecnia y medicina interna.Editorial Lopez Libreros, Buenos Aires, 6ta edición.

31-BARAJAS, J. Las funciones auditivas Centrales. Revista Electrónica de Audiología.2003. Disponible en: www. Auditio. com fecha de acceso: 11 de mayo de 2010.

32- Enciclopedia Salvat Diccionario. Salvat Editores S.A. Barcelona.1972

33-SUROS,J. Semiología Médica Y Técnica Exploratoria.. Salvat Editores. 6ta edición. Barcelona.1978.

34- HUILEY,S, CUMMINGS, S.; BRUWNER W.; GNODY, D ; NEWMAN, T. Diseño de la investigaciones clínicas .Editorial ISBN., Madrid. 3ra. Edición.2008.

35- FLORES, C.; CARRILLO, J.; GRATH, M; HUERTA, R.; ZARATE, T.; MORALES, I. Revista electrónica Nova Scientia. Incidencia de estrés en odontólogos de diferentes especialidades ocasionado por ruido en el consultorio dental. Nro.2, Vol 1 (I).2009.

Texto Traducido por: Fabian Calixto Fraiz

Recibido: 19-03-12

Aceptado: 30-03-12

Correspondencia: teresachirife@gmail.co 